\title{
Escritura femenina y subversión del género en El cuarto mundo, de Diamela Eltit
}

\author{
Female writing and subversion of gender in The fourth world, \\ by Diamela Eltit \\ Eleonora Frenkel Barretto \\ Universidade Federal de Santa Catarina \\ Gisett Lara \\ Universidade Federal de Rio Grande \\ DOI: https://doi.org/10.5902/2176148537328
}

Resumen: Este artículo reflexiona sobre la literatura feminista en el contexto post-golpe militar en Chile, pensándola como aquella que es capaz de cuestionar mecanismos de poder ejercidos desde la masculinidad. La literatura y la performance de Diamela Eltit sobresalen como acciones de intervención y enfrentamiento al control impuesto sobre los cuerpos y, en particular, a la imposición de una bipolaridad de géneros afirmada por una lógica militarista-patriarcal. En El Cuarto mundo, se analiza la subversión a la colonización del género - que impone la heterosexualidad obligatoria y el sometimiento femenino -, a través del travestismo (y la performance de género) y del lesbianismo como gestos de descolonización del cuerpo femenino.

Palabras-clave: Cuerpo. Género. Performance. Colonialismo. Feminismo latinoamericano.

Abstract: This article reflects on feminist literature in the post-military coup context in Chile, thinking it as one that is capable of questioning power mechanisms exercised from masculinity. The literature and performance of Diamela Eltit stand out as actions of intervention and confrontation to the control imposed on the bodies and, in particular, to the imposition of a bipolarity of genders affirmed by a militarist-patriarchal logic. In The Fourth World, the subversion of gender colonization - which imposes compulsory heterosexuality and female subjugation - is analyzed through transvestism (and gender performance) and lesbianism as gestures of decolonization of the female body.

Keywords: Body. Gender. Performance. Colonialism. Latin American Feminism. 
Eleonora Frenkel Barretto

Gisett Lara

El 11 de septiembre de 1973 marca un quiebre histórico y cultural en Chile. Se sustituye al proyecto político socialista, instalado democráticamente por la Unidad Popular, por una dictadura que somete al país durante largos diecisiete años. En dicho periodo, "el ataque a la diferencia fue múltiple e incesante. La escisión entre un nosotros y los otros, puros e impuros, patriotas y extremistas dio inicio al monótono y sostenido binarismo mediante el cual se regimentaron los cuerpos" (ELTIT, 2014, p. 20). Las referencias y símbolos del mundo cultural, a partir de entonces, se vieron imposibilitados de representar tal catástrofe "en circunstancias en que estos lenguajes parecían haber agotado sus energías frente a la tarea de reinventar signos que dieran cuenta de la fractura histórica de memorias e identidades sin rendirse a la violencia desintegrativa de la crisis" (RICHARD, 1993, p. 57).

En ese escenario, "el cuerpo", el mismo que se convirtió en foco político de la dictadura, "trágico territorio modélico de disciplinamiento [...] que se hizo primordial a través de la tortura, el crimen y la desaparición" (ELTIT, 2014, p. 20), fue utilizado como herramienta para desarticular la forma tradicional y dominante de escritura y los discursos hegemónicos de la literatura, constituyendo lo que se denominó literatura post-golpe. ${ }^{1}$ En dicho contexto, ésta "es un cuerpo que revelará, como icono de sí mismo, una escritura, cuyos grafos, la llaga, la exhibición, el hibridismo, serán metáfora que condensará una respuesta al requisado cuerpo nacional" (BRITO, 1994, p. 13). Así, la literatura post-golpe abordó, mediante la literatura, lo que la Escena de Avanzada, a fines de los años 70, hizo a través del arte-experimental; trabajó intensamente sobre la materialidad simbólica de los cuerpos.

La escritura de Diamela Eltit se sitúa entre ambos proyectos. Da inicio a una nueva fase literaria post-golpe ${ }^{2}$-reabriendo un nuevo modelo de

1 Correspondiente a la literatura chilena que emerge a partir del Golpe militar de 1973 y que se encuadra dentro de la llamada literatura de la Resistencia.

2 "Nuevo en el sentido de recreado, retocado por ese indiscutible sello que pactan una lengua y un estilo personal para hacer de un / unos textos una diferencia dentro de la tradición que subvierte, como del lugar que ocupa en la coyuntura literaria post-golpe" (BRITO, 1994, p. 111). 
lectura-escritura- y transformando, a través de la Escena de Avanzada, la literatura en performance, tanto dentro como fuera del texto. A través de la escritura, el cuerpo es utilizado estratégicamente por Eltit, convirtiéndolo en "un espacio desde luego cultural, siempre poblado de signos que hablan del "poder" o lo delatan, en la conceptualización de Foucault, ${ }^{3}$ y de su insistencia secular en colonizar el cuerpo, inscribiendo en él, soterradamente sus códigos" (MORALES, Apud ELTIT, 1997, p. 15). En una primera instancia, Eltit acudió al cuerpo mediante la sexualidad, apelando a la problemática de las identidades. Sin embargo, aquel cuerpo inicial, subvertido -diferente, disidente-, representó también un cuerpo social, "otro" -“cuerpos populares", "subproletarios"- (MORALES, 2014, p. 12).

Nelly Richard, en su obra Feminismo, género y diferencia (2008), en la cual relaciona diferentes obras artísticas realizadas por mujeres du-

Escritura femenina $y$ subversión del género en El cuarto mundo, de Diamela Eltit rante la dictadura militar, analiza, en el ensayo "Diamela Eltit: de la cicatriz al maquillaje", la vinculación entre texto y performance en relación a la obra Lumpérica, junto al trabajo performático de la escritora, realizado en los años en que conformó el grupo CADA (Colectivo de Acciones De Arte). Richard utiliza el "feminismo" como soporte ideológico de las obras desarrolladas por las artistas, ya que éstas instituyen un modo de acción política, que se encuadra con los movimientos de mujeres, en periodo de dictadura, a partir del cual elaboran una oposición teórica a la organización simbólica del pensamiento dominante ${ }^{4}$ y la crítica a la producción seriada, que instaura una nueva estética.

La crítica a las identidades, propuesta por los postulados del feminismo estructuralista y la teoría Queer, sumado al contexto político represivo, principalmente a partir de las dictaduras, ${ }^{5}$ colocaron a muchas feministas tercermundistas frente a nuevos interrogantes, que les han permitido pensar el propio lugar de enunciación y, en función de éste, una serie de estrategias políticas. Preguntas como ¿es "el cuerpo" o "el cuerpo sexuado" la base estable sobre la que operan el género y los sistemas de sexualidad obligatoria? y ¿o acaso "el cuerpo" en sí es articulado por fuerzas políticas a las que les interesa que esté restringido y constituido por las marcas del sexo? estructuraron, por ejemplo, la

3 Leonidas Morales (2014) vincula la concepción del poder de Diamela Eltit con la obra de Foucault porque, incluso, la autora lo ha referido en sus textos críticos.

4 Respecto a los debates sobre identidad y diferencia relacionados al postestructuralismo y a las filosofías de la deconstrucción (RICHARD, 2008, p. 7).

5 En este contexto, las mujeres tercermundistas en Estados Unidos también estaban comenzando a idear estrategias discursivas frente a las identidades. Ver: MORAGA y CASTILLO, 1988. 
Eleonora

Frenkel Barretto

Gisett Lara

reflexión de Judith Butler. Dichos interrogantes constituyen la apertura de "inscripciones corporales, subversiones performativas", uno de los capítulos de la obra El género en disputa: El feminismo y la subversión de la identidad, de Butler, texto con que se instaura y fundamenta la Teoría Queer en 1990, periodo en el que aún la crítica feminista se enfocaba en la diferencia sexual entre hombre y mujer, sin considerar seriamente la existencia de otras sexualidades.

El primer congreso de literatura femenina latinoamericana tuvo lugar en 1987 y abordó, a partir de las Letras, el conjunto de temáticas mencionadas anteriormente. Producido y autogestionado por un grupo de escritoras y críticas chilenas, del cual Diamela Eltit formó parte,${ }^{6}$ el evento fue reconocido como el más importante realizado en periodo de dictadura. Los focos centrales del encuentro fueron la violencia y la censura provocada por la dictadura y el papel periférico de la literatura latinoamericana, frente a los centros hegemónicos del poder; desde donde emergen los discursos académicos oficiales. Ambos cuestionamientos fueron discutidos a partir de dos conceptos fundamentales, específicamente, "mujer" y "literatura", a partir de los cuales fue tratado, por ejemplo, el interrogante ¿tiene sexo la escritura? O, en palabras de Rosario Ferré, “¿Existe, al fin y al cabo, una escritura femenina? ¿Existe una literatura de mujeres radicalmente diferente a la de los hombres?" (FERRÉ, 2003, p. 12).

Nelly Richard plantea asumir como literatura femenina toda aquella que opere cuestionando los mecanismos de poder ejercidos desde la masculinidad. Richard, tomando como referencia el pensamiento de Diamela Eltit, sostiene la utilización de lo femenino como metáfora que permite ampliar las categorías del género, un mecanismo capaz de poner en crisis el signo "mujer", junto a otros de grupos oprimidos por el poder central:

\footnotetext{
Parece necesario acudir al concepto de nombrar como lo femenino aquello que desde los bordes del poder central busque producir una modificación en el tramado monolítico del quehacer literario, más allá que sus cultores sean hombres o mujeres generando creativamente. (RICHARD, 1993, p.36)
}

\footnotetext{
6 A partir del Congreso realizado nace Escribir en los bordes, una compilación de los trabajos de 21 escritoras latinoamericanas. El texto, publicado por Editorial Cuarto Propio, fue presentado por dos de las compiladoras, Eugenia Brito y Carmen Berenguer, quienes realizaron el trabajo en conjunto con Diamela Eltit, Nelly Richard, Raquel Olea y Eliana Ortega.
} 
En 1988, aparece El cuarto mundo, una novela en donde Eltit realiza un recorrido por temáticas que abordan la intimidad de las relaciones familiares, adentrándose en lo más profundo del ser humano. Los personajes principales son una mujer y un hombre, sujetos compuestos, en un inicio, por el famoso binomio femenino-masculino, mellizos que además de dividir protagonismo, dividen la narración de novela. El primer capítulo, "Será irrevocable la derrota," comienza a ser narrado por el mellizo masculino, gestado un día antes que su hermana, en una noche de fiebre materna e impulsos sexuales paternos. El segundo capítulo, "Tengo la mano terriblemente agarrotada", es narrado por la melliza, gestada bajo las mismas condiciones que su hermano. Entre una mezcla de dolor y enfermedad, tendrán que disputar el vientre materno y estar en contacto directo con la intimidad de una mujer que carga

Escritura femenina $y$ subversión del género en El cuarto mundo, de Diamela Eltit consigo el peso del sometimiento femenino en una cultura patriarcal. La voz interna de los personajes principales, vertebra palabra a palabra los sueños, la interioridad y la identidad, elaborando, a partir de la conformación de un "yo" femenino y masculino, una realidad completamente marginal, que se condice con la patria chilena y sudaca ${ }^{7}$.

\footnotetext{
Utilicé ese título, El cuarto mundo, en dos sentidos. Por un lado, habría un cuarto mundo -que sería un mundo más oscuro que habita en cada uno de nosotros-, dentro de lo cultural. Y a su vez, en ese mismo espectro habría una referencia objetiva al Tercer Mundo que habitamos en Latinoamérica. (ELTIT, 1989, p. 1)
}

La utilización del cuerpo dentro de la obra es fundamental; siendo el inicio construido desde el cuerpo materno que gesta -contiene y repele- dos cuerpos, como fue anunciado, hermano y hermana que luchan por un espacio, la mayoría de las veces dominado por el feto masculino. Ambos cuerpos sufren la agonía y el dolor de convivir en un área limitada, donde la hermana es impactada por la presión física y la violencia ejercida por el cuerpo masculino -más fuerte y mayor que ella-, mientras que éste, por su parte, rechaza compartir un lugar que concebía como propio y en función del cual la figura femenina es configurada como una extranjera, una invasora. El cuerpo será utilizado por Eltit como base para la representación de metáforas que no solamente

7 "Sudaca" es un término peyorativo con el que se denomina a los habitantes de América del Sur en España. 
involucran la construcción individual "de sujeto", sino que además se definen a través de lo material y corpóreo, aludiendo a una realidad cultural e histórica en permanente fragmentación, transformándose en un territorio social, familiar y personal de lucha.

El cuerpo cuartomundista habla, rompe, quema, penetra y expele. Son olores y colores animalizados de la carne morena del subde-

Eleonora

Frenkel Barretto

Gisett Lara

sarrollo. El cuerpo de la diferencia que se construye y deconstruye: nacen dos cuerpos, masculino y femenino, en líquidos amnióticos maternales. Son esos mismos cuerpos que luego se transvisten y se penetran. Los límites polares de lo femenino y lo masculino se rompen en la triada de hermanos sin apellido, y casi sin nombres; son ellos, son otros, son nosotros. (GODOY, 2007, p. 1)

Fuera del vientre, la relación entre ambos hermanos toma matices diferentes; desde el rechazo, manifestado constantemente a través del asco de estar juntos, hasta el apego, que les impide estar separados. Ambos crecen imitando las conductas de sus padres, quienes, por su parte, mantienen una relación dependiente y opresiva. Con el nacimiento de una tercera hija -definida como andrógina y llamada de "María de Alava"- la historia se fragmenta aún más y se convierte en una lucha de poderes. María de Alava representa la ambigüedad de un género que se vincula con el modelo patriarcal a partir de la similitud y afinidad que comparte con el padre.

La melliza, por su lado, encarna una feminidad conflictiva, a veces dominante y otras, sumisa. Constituye la otra parte de una unidad formada por ella y su hermano, quien, en el discurrir de la trama, se torna su compañero y amante, y con el cual, da a luz una niña sudaca, que lleva por nombre diamela eltit (con minúscula). Cuando Eltit comenzó a escribir $E l$ cuarto mundo acababa de terminar el proceso de Lumpérica (1983), una novela considerada complicada, ante lo cual reconoció como una necesidad tranquilizar su prosa; aun así, comenzó escribiendo a partir de la melliza, lo que, según ella, resultó ser bastante "cifrado y oscuro" (ELTIT, 1995, p. 345) por lo que, después de modificaciones, ese texto, resultó ser la voz masculina que relata la primera parte de la novela.

La subversión del género es elaborada en El cuarto mundo, principalmente, a través de la construcción y transformación del hermano mellizo. Diamela Eltit divide su obra, no solo alternando el uso de narradores entre la primera y la segunda parte, sino, también, al recrear 
la voz masculina del primer capítulo, asumiendo desde la ficción una postura "masculina", intentando coincidir con la "racionalidad" tradicionalmente adjudicada a los hombres:

Escribí la primera parte como una "señorita" con una sintaxis más o menos académica y con una historia perceptible y para eso fue necesario en ese momento para la novela tomar una rama masculina que fue el poder de la sintaxis, algo más dominante, más tradicional. Me salió tan fluidamente que me pareció que había algo masculino en mí (ELTIT, 1995, p. 345).

Cuando Diamela reconoce que desconocía que algo masculino había en ella, asume su literatura desde la feminidad autoafirmada, lo que implica el desplazamiento de los signos. Eltit crea una voz masculina que coincide con el modelo de "razón", asignada tradicionalmente al hombre, sin embargo, asume, posteriormente, una voz femenina, que responde en algunos aspectos al modelo de feminidad patriarcal, es decir, que representa caos e interioridad, superior en términos de complejidad del discurso.

Na ficção e no risco que porta a atividade de pensar, atrevo-me a propor aqui, como jogo literário, a mulher-costela. Ela me interessa (por enquanto) devido a sua própria emergência, e me parece atrativa pelo seu hiperfantasioso traço, que hoje pode ser visto como uma superlativa estória de ficção científica, ou como filme em três dimensões. Mas também como sofisticada fotografia, uma figura que já contém, na ficção de sua arquitetura, os signos poéticos e, a partir daí, o trabalho social deita raízes em politizar essas marcas, estabelecer nó certeiro de signos políticos para emancipar-se de sua própria padronização (ELTIT, 2017). ${ }^{8}$

El discurso de la melliza es un discurso eminentemente político, que trasciende al "yo" presentado por el hermano mellizo, cuyo límite fue realizar la génesis de la familia sudaca. Es a partir del segundo capítulo donde aparece el compromiso con "la nación degradada", en otras

8 La cita se encuentra en el ensayo "Com a cultura nas mãos: a mulher, a costela e os signos", publicado en A máquina Pinochet e outros ensaios, lanzado únicamente en portugués, y carece, en esta ocasión, de referencia de página, al haber sido el acceso a dicho libro solo a través del formato epub. 
palabras, la necesidad de establecer un pacto político desde la "fraternidad sudaca". Este discurso de la melliza está mezclado con el dominio que posee de su propia sexualidad, de su cuerpo, un discurso que le otorga, al mismo tiempo, distancia de la familia. La sitúa como gestora de un proyecto contra hegemónico, materializado en la hija, quien, al mismo tiempo, deberá desafiar cualquier indicio de tradición de un linaje materno atravesado por el sistema patriarcal:

Eleonora

Frenkel Barretto

Gisett Lara

174

Los pechos hinchados. El dolor de la leche. El niño, en complicidad con el resto de la familia, me ataca desde dentro. He incubado a otro enemigo y sólo yo conozco la magnitud de su odio. Mi cabeza. En mi cabeza se gestan sueños confusos, recorridos por vastos campos de dudas. Las dudas del niño pasean por mi cerebro grávido. Aprendo, a través del dolor, a conocer todos los rincones de mi cuerpo y la furia orgánica con que se ejerce el castigo (ELTIT, 1996, p. 236).

De María Chipia, solamente es posible saber, en el primer capítulo narrado por él, el origen de su nombre femenino, que representa la venganza de la madre al padre, es decir, al poder masculino. Si bien, oficialmente al mellizo se le asigna el nombre del padre, la madre usurpa la ceremonia del denominado "rito sacro" y lo define como María Chipia:

\footnotetext{
Se me otorgó el nombre de mi padre. A mi hermana se le designó también un nombre. Mi madre, solapadamente, me miró y dijo que yo era igual a María Chipia, que yo era ella. Su mano afilada recorrió mi cara y dijo: “Tu eres María Chipia” (ELTIT, 1996, p. 158).
}

El nombre enunciado por la madre marca simbólicamente la frontera sexual de una masculinidad que más tarde será subvertida por la influencia femenina de su hermana melliza. De esta manera, María Chipia irrumpe, en el segundo capítulo de la obra, bajo la narración de su hermana melliza. Es la voz de la melliza diamela eltit -alter ego de la escritora- quien le otorga identidad a su hermano desde la feminidad. “Tengo la mano terriblemente agarrotada", título que indica la apertura de esa segunda parte de la novela, comienza con la descripción de la escena sexual entre ambos -hermana y hermano-. 
Mi hermano mellizo adoptó el nombre de María Chipia y se travistió en virgen. Como una virgen me anunció la escena del parto. Me la anunció. Me la anunció. La proclamó (ELTIT, 1996, p. 211).

Diamela Eltit utiliza la figura de la travesti para quebrar, mediante el artificio del género, el aspecto visible de la masculinidad. En relación a este aspecto, vale destacar que, desde el terreno artístico y literario en Chile, durante el periodo de dictadura, la figura de la travesti fue un recurso altamente utilizado, ya que, reúne o anula dos polaridades, que implican la dominación y el sometimiento. Su imagen representa la trasgresión tradicional del binarismo femenino-masculino que, en dicho contexto histórico, simbolizó la división entre la lógica militarista-patriarcal de la dictadura y la democracia sometida, cuyo

Escritura femenina $y$ subversión del género en El cuarto mundo, de Diamela Eltit rostro visible era el pueblo vulnerado y reducido al espacio doméstico, tradicionalmente asignado a la mujer:

\footnotetext{
La figura del travesti chileno que emerge durante estos años de los clandestinajes del deseo reprimido en la sombra más tortuosa de los códigos de regimentación del sentido, es la figura que socava el doble ordenamiento de esa masculinidad y femineidad reglamentarias y de fachada (RICHARD, 1993, p. 65).
}

Diamela Eltit utiliza la figura de la virgen para escenificar la performance de María Chipia. La virgen-madre, representación del modelo cultural y religioso dominante, es suplantada por una virgen "malinchezca". Proceso que se produce cuando "pisoteando a su virgen que llamaba al mal, que significaba el mal, María Chipia se dobló en el suelo y su boca mordió el polvo. Desnudo, como hijo de Dios. Me debatí en la mancha de sangre. Iracunda, como hija de Dios" (ELTIT, 1996, p. 211).

En El cuarto mundo, es la madre de la familia sudaca quien carga con la culpa y el sometimiento del modelo mariano occidental. En el primer capítulo el hijo sufre, a través de su madre, el padecimiento de la violación del padre: "Ese 7 de abril fui engendrado en medio de la fiebre de mi madre y debí compartir su sueño. Sufrí la terrible acometida de los terrores femeninos" (ELTIT, 1996, p. 147). El sufrimiento, la depresión y el constante deseo de inexistencia que vive la madre surgen, en la narrativa, a partir de la relación conyugal. Contribuyen a la interpretación de este pasaje la lectura de Sonia Montecino, quien analiza 
el "culto a la apariencia" en la sociedad chilena, a partir de la idea de matrimonio obligado o una falsa nulidad, vinculándolo con la característica de la sociedad mestiza que utiliza las apariencias como "disfraz" o "máscara". 9 Lo que explica que temas como "divorcio" o "aborto" sean evadidos del discurso público, intentando resguardar la familia, o la apariencia de familia, ya que en la práctica existen ambas realidades, encubiertas discursivamente:

Eleonora

Frenkel Barretto

Gisett Lara

176

Por eso, creemos que una serie de demandas -que son comunes en las sociedades "modernas"- atingentes al ámbito de la familia y de la sexualidad, por ejemplo, encuentren en nuestros territorios serias dificultades para su enunciación y para su realización (MONTECINO, 1998, p. 110-111).

La renuncia de María Chipia al modelo mariano constituye el rechazo al modelo femenino de la madre, desde la feminidad que le otorga el travestimento, $y$, también es la oposición al mandato de masculinidad que como travesti representa la renuncia al padre, la negación a seguir perpetuando el modelo de hombre "macho" del proyecto colonial y patriarcal:

En este contexto, el de la reformulación travesti textual, el propio performance identitario transgrede y cuestiona las marcas patriarcales y los dominios de clase que moldean y reproducen la cultura chilena contemporánea dentro de su formato capitalista que hoy conserva sus legados más arcaicos y autoritarios (LLANOS, 2010, p. 147).

Diamela Eltit utiliza la figura de la travesti para fecundar en la melliza, mestiza, la continuidad del proyecto iniciado desde la fraternidad sudaca. En este sentido, la identidad femenina del hermano se materializa en María Chipia, quien genitalmente cumple con la función masculina necesaria para la reproducción, sin embargo, su esencia ha dejado de ser masculina: "Al ignorar la jerarquía tradicional entre apariencia y esencia, interior y exterior, realidad y simulación [...] adquiere una desconocida movilidad, libre finalmente de las restricciones artificiales [...] que le im-

9 Siguiendo el pensamiento de Octavio Paz (1959) respecto a la configuración de la identidad mestiza en México. 
pone la cultura dominante" (RICHARD, 1993, p. 73). En la novela de Eltit, la madre preconiza: "Divino. Divino. El ego maltrecho de María Chipia. La herida ranural de las mujeres" (ELTIT, 1996, p. 218).

\begin{abstract}
Cuando yo me maquillo elijo ocultar parte de mí y la varío para develar o fingir(me) otra. También telas y vestuario colaboran en a mi cambio y me encubren vistiéndome. La cosmética y la moda son alteraciones pasajeras, así como fugaz y transitorio es el pasaje logrado por el hombre que quiere $y$, solo por un breve instante, puede ser mujer (BIANCHI, 2012, p. 10-11).
\end{abstract}

A través de la transformación de María Chipia es posible percibir que la relación sexual con su hermana corresponde simbólicamente a una relación homosexual entre figuras o representaciones femeninas. Sobre esto, Eltit indica: "quise jugar con las imposibilidades; que el protagonista, por ejemplo, quebrara su estatuto sexual, que fuera más ambiguo [...] está quebrando su identificación inicial ya por maquillaje, ya por sensibilidades" (ELTIT, 1995, p. 346). Este hecho no solo constituye la anulación de la masculinidad patriarcal -mediante el quebrantamiento de la heterosexualidad obligatoria-, sino también, la instauración de un pacto político desde lo femenino -la fraternidad sudaca-, concretizada a partir del acto sexual entre hermanos, de cuya unión nace una niña sudaca.

A partir de la subversión del género, de María Chipia y de su hermana melliza, es posible incorporar el lesbianismo como contribución epistémica de descolonización del cuerpo femenino, aspecto que contribuye a repensar el papel de las comunidades latinoamericanas, formadas entre hombres y mujeres. Las contribuciones del lesbianismo en el análisis feminista otorgan distanciamiento a la práctica heterosexual que ha sido impuesta y naturalizada desde la colonización, en donde “así como la fundación del capitalismo occidental dependió del tráfico de esclavos en el Atlántico Norte, el sistema de la dominación patriarcal se sostiene en la sujeción de las mujeres a través de una heterosexualidad obligatoria" (CLARKE, 1988, p. 101).

En este sentido, el lesbianismo funciona como puente de autoafirmación femenina que permite visualizar la conexión, las relaciones y las organizaciones entre mujeres, distanciándose del poder masculino ejercido por el hombre. No es casual que "muchas de las primeras mu-

\section{Escritura \\ femenina $y$ subversión del género en El cuarto mundo, de Diamela Eltit}


jeres de color que se identificaron como feministas eran lesbianas. Su propia identificación lésbica les hacía imposible la postergación de sus intereses como mujer" (MORAGA, 1988, p. 3). Aquella identificación lésbica permite crear un lugar de resistencia a la colonización del género $\mathrm{y}$, a partir de ese espacio, construir diálogos horizontales en las comunidades donde lo femenino y masculino funcione de forma complementaria y no excluyente.

Eleonora Ochy Curiel, al analizar el papel del feminismo latinoamericano, Frenkel Barretto

Gisett Lara realiza una breve exposición sobre las prácticas descolonizadoras instauradas en el continente, cuyo origen se encuentra precisamente "en feministas racializadas, en las lesbianas, en mujeres del 'Tercer Mundo', aquellas que se resistieron a la dominación patriarcal, racista y hetero178 sexista desde posiciones subalternas" (CURIEL, 2010, p. 70), y sobre los desafíos que emergen a partir de esta práctica, que no es solo teórica, sino, fundamentalmente, activista. En este proyecto descolonizador resulta vital la incorporación de las voces de mujeres, cuyas "posiciones feministas" e "indígenas" han resistido al embate colonial (MARCOS, 2013, p. 145).

Julieta Paredes, por su parte, denuncia la influencia del patriarcado occidental sobre el movimiento indigenista ${ }^{10} \mathrm{y}$ la estructuración machista de las políticas bolivianas a partir del concepto de "chacha-warmi" (hombre-mujer). ${ }^{11}$ El término es utilizado en la cosmovisión andina, definido como un código de conducta basado en el principio de dualidad y complementariedad, empleado, además, por el gobierno de Bolivia para impulsar las políticas de descolonización y despatriarcalización. Es reconocido, en la actualidad, como equidad entre los géneros. A pesar de que este concepto, teóricamente, comprende a la mujer como una de las partes fundantes de la dualidad del ser humano, su importancia está supeditada al hombre. Un ejemplo, expuesto por Paredes, reside en que solo los hombres son elegidos para funciones políticas, mientras que las mujeres, que constituyen la otra mitad, son sus esposas, y no candidatas igualmente electas por el pueblo, quedando el protagonismo femenino reducido, nuevamente, a la esfera familiar y privada (PAREDES, 2008, p. 8).

10 El movimiento indigenista en Bolivia existe desde la colonia. Durante el gobierno de Evo Morales, tuvieron mayor injerencia en el Estado.

11 Chacha-warmi es traducido como hombre-mujer desde la visión occidental, sin embargo, para el pueblo aymara, no son estructuras rígidas. Es posible performar "chacha" o "warmi" dependiendo de múltiples factores. 
En El cuarto mundo, si bien hermana y hermano se relacionan de forma jerárquica, mediante representaciones aprendidas de sus padres, el compartir el mismo espacio uterino crea conexiones entre ambos que, más tarde, cuando superen la división jerárquica y establezcan un pacto político, surge la necesidad de volver a este inicio, al origen, que es metáfora, también, del origen latinoamericano percibido como dualidad entre femenino y masculino, lo que, en la novela de Eltit, aparece recurrentemente en los sueños de los personajes. Un mundo onírico, en el que las figuras de animales se unen en un signo fraternal:

\footnotetext{
En mis sueños volvían a aparecer esas dos figuras amalgamadas que se trenzaban en un abrazo o en una lucha, debatidas en la calidez de las aguas. Hube de responder a la voracidad de esas imágenes y me preparé a enfrentarme a ella tal como un amante en su primera cita (ELTIT, 1996, p. 193).
}

La complementariedad entre lo femenino y masculino, propuesta por Eltit, corresponde efectivamente a la compresión del "chacha-warmi" como organismo complementario, más allá de las categorías hombre-mujer que promueve el movimiento indigenista, aludiendo exclusivamente a la relación de pareja heterosexual, ocultando la importancia de la participación de las mujeres en el cuerpo de la comunidad. La comprensión limitada del "chacha-warmi" ha fomentado el rechazo de otro tipo de vínculos en Bolivia, existiendo gran resistencia, tanto a las relaciones homosexuales como de los movimientos definidos como feministas (PAREDES, 2008, p. 9):

Entre las feministas comunitarias hay pensadoras que se han separado de su comunidad sin renunciar a ella y que ejercen su sexualidad confrontando la moralidad impuesta, evidenciando la presencia histórica de las lesbianas indígenas. Son teóricas del feminismo comunitario que carean estética, política, económica y culturalmente los constructos opresivos de los roles sexuales, negándose a asumir como indígenas una identidad blanqueada en las ciudades donde se han asentado por motivos de migración familiar, de estudio o de trabajo, a la vez que como feministas exigen cultivar una vida pensada desde la autonomía de movimiento y de opinión (GARGALLO, 2014, p. 296). 
La importancia de cuestionar la heterosexualidad obligatoria desde los movimientos de mujeres feministas indígenas radica en la desestructuración de la imagen estereotipada de los propios pueblos, de las comunidades, $\mathrm{y}$, principalmente, del modelo de mujer indígena construido dentro de la lógica occidental, donde ser indígena y lesbiana no es compatible. A partir de estas demandas es posible visualizar las potencialidades de un discurso que intenta desmantelar la lógica patriarcal, incluso, dentro de las

Eleonora mismas comunidades indígenas. La subversión del género implica el rechaFrenkel Barretto

Gisett Lara zo a la imposición que define la identidad y controla los cuerpos a través de la sexualidad. En ese sentido: "Históricamente el pensamiento lésbico ha sido un lugar de escondite y de exposición de un proyecto distinto de sociedad, donde no se necesita de la tolerancia de los poderes económicos, religiosos, culturales y políticos para existir" (PISANO, 2004, p. 91).

En este sentido, retomar el trabajo de Diamela Eltit permite reivindicar un proyecto político y literario iniciado hace más de treinta años. Constituye un puente entre las mujeres tercermundistas que nos conecta con la fuerza autoafirmada de la mestiza que carga y equilibra los signos de ambas culturas creando una nueva consciencia. Eltit inscribe, en el cuerpo textual y sexual, nuevas formas de resistir, desestructurando la normatividad del sistema que nos aprisiona. La subversión de la "colonialidad del género" permite la autoafirmación de la mujer latinoamericana y, por consiguiente, la recuperación de su poder político en las comunidades.

\section{REFERÊNCIAS}

BIANCHI, Soledad. Maquillaje para una carcajada triste. In: CASAS, Francisco. Sodoma MÍA. Santiago: Pequeño Dios Editores, 2012.

BRITO, Eugenia. Campos Minados (Literatura post-golpe en Chile). Santiago: Cuarto Propio, 1994.

BUTLER, Judith. Problemas de gênero: feminismo e subversão da identidade. Tradução de Renato Aguiar. Rio de Janeiro: Editora Civilização Brasileira, 2003.

CASAS, Francisco. Sodoma MÍA. Santiago: Pequeño Dios Editores, 2012. 
CLARKE, Cheryl. El lesbianismo un acto de resistencia. In: MORAGA, C. y CASTILLO, A. Esta puente, mi espalda. Voces de mujeres tercermundistas en los Estados Unidos. San Francisco: Editorial “ismo”, 1988.

CURIEL, Ochy. Hacia la construcción de un feminismo descolonizado. Escritura In: ESPINOSA MIÑOSO, Yuderkys. Aproximaciones críticas a las femenina $y$ prácticas teóricas políticas del feminismo latinoamericano. Buenos Aires: En La Frontera, 2010. subversión del género en El cuarto ELTIT, Diamela. Lumpérica. Santiago: Ornitorrinco, 1983. mundo, de Diamela Eltit

ELTIT, Diamela. El trabajo es la vida y mi vida. El Mercurio, Santiago, p.1, 21 mayo, 1989. Entrevista concedida a Larraín, Ana María.

ELTIT, Diamela. L. Iluminada en sus ficciones: Conversación con Diamela Eltit. Revista de Literatura Hispánica, University of Connecticut, no 40-41, pp. 335-366, 1995. Entrevista concedida a BURGOS, Fernando y FENWICK, M.J.

ELTIT, Diamela. El Cuarto Mundo. Santiago: Seix Barral, 1996 (1a edición de 1988).

ELTIT, Diamela. Narración y referentes en Diamela Eltit. Revista Chilena de Literatura, Santiago, nº 51, pp. 121-130, 1997. Entrevista concedida a MORALES, Leonidas.

ELTIT, Diamela. Emergencias: escritos sobre literatura, arte y política. Santiago: Seix Barral, 2014.

ELTIT, Diamela. A máquina Pinochet e outros ensaios. São Paulo: e-galaxia, 2017.

FERRÉ, Rosario. La cocina de la escritura. Argentina: Biblioteca virtual universal, 2003.

GARGALLO, Francesca. Feminismos desde Abya-Yala. Ideas y proposiciones de las mujeres de 607 pueblos en nuestra América. Ciudad de México: Corte y confección, 2014. 
LLANOS, Bernardita. Esas locas madres de Pedro Lemebel. In: BLANCO, Fernando. Desdén al infortunio. Sujeto, comunicación y público en la narrativa de Pedro Lemebel. Santiago: Cuarto Propio, 2010.

MARCOS, Sylvia. Descolonizando el feminismo: la insurrección epistemológica de la diferencia. In: TORRES, Georgina et al. Senti-pensar el género: perspectivas desde los pueblos originarios.

Eleonora En Senti-pensar el género. México: Casa del mago, 2013.

Frenkel Barretto

Gisett Lara

MONTECINO, Sonia. Madres y huachos: alegorías del mestizaje chileno. Santiago: Anagrama, 1998.

MORALES, Leonidas. El discurso crítico de Diamela Eltit: cuerpo y política. In: ELTIT, Diamela. Emergencias: escritos sobre literatura, arte y política. Santiago: Seix Barral, 2014.

MORAGA, Cherríe. y CASTILLO, Ana. Esta puente, mi espalda. Voces de mujeres tercermundistas en los Estados Unidos. San Francisco:Editorial “ismo”, 1988.

MORAGA, Cherríe. En el sueño, siempre se me recibe en el río. In: MORAGA, C. y CASTILLO, A. Esta puente, mi espalda. Voces de mujeres tercermundistas en los Estados Unidos. San Francisco: Editorial “ismo”, 1988.

PAZ, Octavio. El laberinto de la soledad. México: Fondo de Cultura Económica, 1959.

PAREDES, Julieta. Hilando fino desde el feminismo comunitario. La Paz: El Rebozo, 2008.

PISANO, Margarita. El triunfo de la masculinidad. Fem-e-libros, 2004.

RICHARD, Nelly. Femenino / Masculino: prácticas de la diferencia y cultura democrática. Santiago: Francisco Zegers, 1993.

RICHARD, Nelly. Feminismos, género y diferencias. Santiago: Palinodia, 2008. 